FRACTIONAL QUANTUM HALL EFFECT

\title{
Full tilt
}

An in-plane magnetic field usually destroys the isotropic fractional quantum Hall states of two-dimensional electron systems, and gives rise to anisotropic liquid-crystal-like states. An unexpected observation of the coexistence of both states at once suggests the emergence of a new quantum phase of matter.

\section{Chetan Nayak}

$\mathrm{T}$ he fractional quantum Hall effect (FQHE) is a manifestation of a remarkable state of matter that occurs when electrons are confined within a high-mobility two-dimensional electron system (such as a semiconductor interface or quantum well) and subjected to low temperatures and high perpendicular magnetic fields ${ }^{1}$. As the name suggests, this state is characterized by fractionally quantized Hall resistance and zero longitudinal resistance, arising from the formation of fractionally charged quasiparticles consisting of cooperative excitations of electrons. At low fields, a qualitatively different 'quantum Hall nematic state' can emerge ${ }^{2-5}$. Despite the name, this state is not precisely quantized but is exotic nonetheless, in that it exhibits anisotropy that bears no clear relation to the properties or structure of the crystal lattice that supports it. It was widely believed that these two states were mutually exclusive, as decreasing the perpendicular field or even, in one case, simply increasing the inplane field destroys the FQHE and replaces it with the nematic state. But writing in Nature Physics ${ }^{6}$, Xia and colleagues report that when they tilt a two-dimensional electron system with respect to a strong, initially perpendicular magnetic field the Hall resistance at filling fraction $v=7 / 3$ remains quantized (the hallmark of the FQHE), but becomes markedly anisotropic (the hallmark of the nematic state) suggesting that these two states can, in fact, coexist.

To understand why this is so surprising, it is important to first note that a fractional quantum Hall state has an energy gap, below which there are no mobile excitations. Consequently, at temperatures much lower than the gap, the longitudinal resistance falls to zero with decreasing temperature. In contrast, in a metal, excitations persist at all energies above the ground state. And so it is difficult to see how a system could simultaneously exhibit the FQHE and metallic longitudinal resistance. The contradiction is even more acute in the behaviour that Xia et al. observe: not only do mobile excitations persist at even the lowest temperatures, but their mobility increases in one direction with decreasing temperature and, consequently, the resistance increases in the perpendicular direction, which Xia et al. therefore call the 'hard' direction.

Of course, it is possible that the longitudinal resistance in the 'hard' direction might also turn around and begin to similarly decrease at temperatures below those reached by the authors, so that both vanish as the classic FQHE suggests they should. In this case, the nematic behaviour would represent an intermediate-temperature phenomenon. It is also worth noting that the electrons do not choose a direction entirely on their own - when the device is tilted, the in-plane component of the magnetic field points in a particular direction in the plane. So a sceptic might say that the anisotropic longitudinal resistances are simply a transient phenomenon caused by a large in-plane magnetic field.

But, the increase of the longitudinal resistance in the hard direction with decreasing temperature is sudden and dramatic. Furthermore, the longitudinal resistance in this direction is large enough at the lowest temperatures obtained by Xia et al. (approximately $15 \mathrm{mK}$ ) that the FQHE should not be observed - mobile excitations should cause the Hall resistance to vary from its quantized value. Finally, the system seems to become isotropic again at a tilt of 76 degrees. This interpretation of the data is made more plausible by the fact that at the nearby $v=5 / 2$ plateau the authors found that small tilts destroy the plateau, enabling a quantum Hall nematic state to emerge, but larger tilts drive the system into an isotropic metallic state similar to that seen at $v=1 / 2$ (ref. 7 ).

Thus, it is plausible that the electrons would spontaneously choose a direction on their own if the in-plane field didn't do it for them. Then, the onset of anisotropy could be a would-be phase transition into a phase in which nematic order could coexist with the FQHE, as suggested by some theories ${ }^{8-10}$. This could be compatible with an eventual decrease of both resistivities at the lowest temperatures, because the effects of nematic symmetry breaking might only manifest themselves at intermediate temperatures. Alternatively, Xia et al. suggest their results could be the result of an inhomogeneous phase in which some regions are in a fractional quantum Hall state while others are in an insulating state. If these different regions were to form alternating stripes with a preferred direction, the longitudinal resistances would be highly-anisotropic, as observed. But, it is difficult to see how the Hall resistance would be quantized, as it would necessarily involve contributions from both phases.

Regardless of the explanation, the results demonstrate the coexistence not only of state but of behaviour, topological ordering (the underlying ordering that characterizes the FQHE) and symmetry breaking (the more conventional ordering that characterizes most known phases of matter). Further proof, if any were needed, of the rich behaviour that can emerge as a result of the self-organization of electrons under extreme conditions.

Chetan Nayak is at Microsoft Research, Station Q and in the Department of Physics, University of California, Santa Barbara, California 93106-6105, USA.

e-mail: nayak@kitp.ucsb.edu

\section{References}

1. Prange, R. \& Girvin, S. M. (eds) The Quantum Hall Effect (Springer, 1986)

2. Lilly, M. P., Cooper, K. B., Eisenstein, J. P., Pfeiffer, L. N. \& West, K. W. Phys. Rev. Lett. 82, 394-397 (1999).

3. Du, R. R. et al. Solid State Commun. 109, 389-394 (1999).

4. Koulakov, A. A., Fogler, M. M. \& Shklovskii, B. I. Phys. Rev. Lett. 76, 499-502 (1996)

5. Fradkin, E. \& Kivelson, S. A. Phys. Rev. B 59, 8065-8072 (1999).

6. Xia, J., Eisenstein, J. P., Pfeiffer, L. N. \& West, K. W. Nature Phys. 7, 845-848 (2011).

7. Xia, J., Cvicek, V., Eisenstein, J. P., Pfeiffer, L. N. \& West, K. W. Phys. Rev. Lett. 105, 176807 (2010).

8. Musaelian, K. \& Joynt, R. J. Phys. Cond. Matt. 8, L105-L110 (1996).

9. Mulligan, M., Nayak, C. \& Kachru, S. Phys. Rev. B 82, $085102(2010)$

10. Mulligan, M., Nayak, C. \& Kachru, S. http://arxiv.org/abs/1104.0256 (2011).

Published online: 23 October 2011 